Published in: Sensors and Actuators B 181 (2013) 71-76

\title{
Polyethylene imine-based receptor immobilization for label free bioassays
}

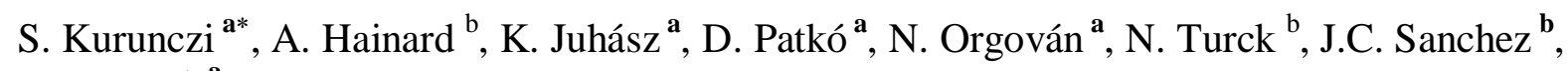
R. Horvath ${ }^{\text {a }}$

${ }^{a}$ Department of Photonics, Institute for Technical Physics and Materials Science, Research

Centre for Natural Sciences (MTA TTK MFA), H-1121, Konkoly Thege Miklós út 29-33,

Budapest, Hungary

${ }^{\mathbf{b}}$ Department of Human Protein Sciences, Medical University Center, Geneva University, Geneva, Switzerland

\begin{abstract}
Polyethylene imine (PEI) based immobilization of antibodies is described and the concept is proved on the label free assay of C-Reactive Protein (CRP). This novel immobilization method is composed of a hyperbranched PEI layer which was deposited at a high pH (9.5) on the sensor surface. The free amino groups of PEI were derivatized with neutravidin by Biotin $\mathrm{N}$-hydroxysuccinimide ester and the biotinylated anti-CRP antibody immobilized on this layer. Direct binding assay of recombinant CRP was successfully performed in the low $\mu \mathrm{g} / \mathrm{ml}$ concentrations using a label free optical waveguide biosensor.
\end{abstract}

Keywords: room temperature immobilization, polyethylene imine (PEI), label free sensor, neutravidin, C-reactive protein (CRP), OWLS

\section{Introduction}

An important aspect of biosensor development is the biofunctionalization protocol $[1 ; 2]$. Conventional immobilization methods are based on silanization of sensor surfaces with amino

\footnotetext{
* Corresponding author. Tel.: +36 1392 2696, Fax: +36 13922226

E-mail address: kurunczi.sandor@ttk.mta.hu (S. Kurunczi)
} 
(or epoxy, thiol) functional silanes and grafting the receptor molecules (typically antibodies) to the silanized surface by a cross-linker, such as EDC/NHS (or glutaradehyde, BS3, etc.) [3]. Except the well-known dextran layer in SPR applications [4], most detection assays rely on monolayer immobilization methods to graft antibodies to surfaces setting an ultimate limit to the surface coverage of receptor molecules. There is a need for more efficient immobilization methods with potential simplification of this multistep preparative work. It is especially the case when emerging technologies with disposable biochips are considered [5; 6]. This contribution focuses on the design of an antibody containing graft architecture (composed of polyethylene imine, PEI) that could be used for biosensor surface preparation. In this work a table top biosensor was used to follow the building up of the molecular layers. Optical waveguide lightmode spectroscopy (OWLS) is a surface sensitive biosensor using evanescent waves. The technique was successfully applied to monitor lipid bilayers, protein adsorption or even living cells. [7; 8; 9].

Pre-adsorbed polyelectrolyte layers are advantageously used in various applications from biosensors to basic physico-chemical studies of interfaces [10;11]. In such applications the utility of polyethylene imine is mainly to control the surface potential as PEI bears a number of ionizable amino-groups which possesses positive charge with a density depending on the acidity of the environment. Recently [12] used PEI for direct antibody immobilization for the preparation of neuron-adhesive coatings. Our aim was to use the PEI, a cationic polyelectrolyte adsorbed layer as a simple and robust method for surface functionalization with amino groups. The most common and wide-spread surface amination is based on silane reagents for the silica-type surfaces and for the OWLS sensing surface as well [13; 14]. Silanization is normally completed by a high temperature curing on the sensing surface, a potential drawback in case of plastic made sensor chips $[5 ; 6 ; 15]$.

The biotin binding proteins (avidin, streptavidin, neutravidin) are from a family of proteins presenting a high affinity and selectivity for biotin. This property permits to bridge a surface coated with avidin and the biotinylated antibody. The system presents a double advantage: an appropriate orientation of antibodies (if biotin is bound to their Fc fragments) and a high affinity of immobilization. Among various technologies being used for linking functional groups to bio-molecules, the avidin/biotin noncovalent linkage has attracted significant focus due to the simplicity and versatility of this system [16; 17]. Many proteins can be conveniently biotinylated, as in the case of antibodies (Immunoglobulines; Ig). In this work biotinylated anti-CRP antibody (sheep IgG) was obtained from commercial resource. CRP has been acknowledged as a possible predictor of the risk of an imminent stroke. It has been 
reliably observed that superior concentrations of CRP are related with larger brain infarcts and poor neurologic outcome. Whether a diminution of CRP quantity could be favourable to stroke patients remains to be elucidated [18]. As part of our project, we selected CRP recognition as a convenient way to demonstrate the immobilization chemistry under development. ELISA workflows were used to evaluate antibody-antigen integrity and affinity. There is a need for advanced label-free detection of biomarkers, and for the case of CRP a sensitivity of $0.1 \mu \mathrm{g} / \mathrm{ml}$ was reported after signal enhancement using NHS-dextran surface chemistry in SPR biosensor [19].

The strategy of this interface chemistry was inspired by a previous work [20], where the researchers built up multilayers by repeated deposition of avidin and biotinylated polymers. Such avidin-polymer layers have not been applied in biosensors to the best of our knowledge. Our proposed immobilization layer is composed of a surface adsorbed PEI (crosslinked by glutaraldehyde), functionalized with a biotin-linker, affinity bound neutravidin and finally the biotinylated IgG as shown in Fig 1. The proposed PEI-biotin-neutravidin layer is a monolayer, however possesses an extended molecular structure originating from the hyperbranched PEI molecular structure at basic $\mathrm{pH}$.

\section{Figure 1.}

\section{Material and methods}

Branched PEI with a molecular weight of $750 \mathrm{kDa}$ purchased from Sigma-Aldrich, was used as obtained. Biotin N-hydroxysuccinimide ester (NHS-biotin) was obtained from SigmaAldrich and was dissolved in dimethylformamide, stored at $-20{ }^{\circ} \mathrm{C}$ in aliquots until use. Phosphate Buffered Saline (PBS) tablets have been obtained also from Sigma-Aldrich which yield a $10 \mathrm{mM}$ phosphate buffer containing $27 \mathrm{mM}$ potassium chloride and $137 \mathrm{mM}$ sodium

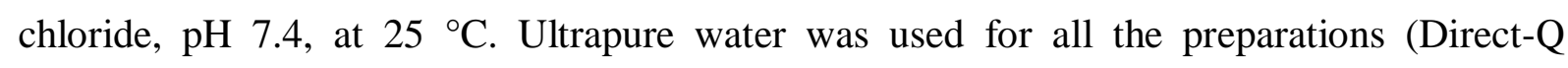
system). Borate buffer (12.5 mM) was prepared from borax and sodium hydroxide to give $\mathrm{pH}$ 9.5 solution.

CRP - Recombinant human C-Reactive Protein (CRP) was obtained from R\&D systems. Polyclonal sheep IgGs against human CRP unconjugated and conjugated with biotin were purchased from R\&D Systems. All these products were received lyophilized and they were reconstituted according to the manufacturer's instructions. Rabbit anti-sheep secondary 
antibody conjugated with alkaline phosphatase (AP) were purchased from Abcam. Streptavidin conjugated with AP as well as AttoPhos ${ }^{\circledR}$ AP Fluorescent Substrate were obtained from Promega. Non-treated 96-well plate (black, polystyrene) as well as neutravidin High Binding Capacity Coated Plates (black 96-well) were obtained respectively from PerkinElmer and Pierce. HSCD7 buffer (DB, polyvinyl alcohol, 80\% hydrolyzed, Mr 9000-10 000 (Aldrich, Milwaukee, WI, USA), MOPS (Sigma), $\mathrm{NaCl}, \mathrm{MgCl} 2$ (Sigma), ZnCl2 (Aldrich), pH 6.90, BSA 30\% solution, manufacturing grade (Serological Proteins, Kankakee, IL, USA)) were freshly prepared.

\section{CRP antibody-antigen integrity and affinity evaluation by ELISA}

\section{Biotinylated anti-CRP antibody coating}

ELISA was performed on commercially available neutravidin coated 96-well plates. Plate wells were pre-wet with $50 \mu 1$ of borate buffer and then $50 \mu 1$ of biotinylated anti-CRP antibody diluted in HSCD7 buffer were assayed at seven different concentrations $(2000,800$, 320, 128, 51.2, 20.5 and $8.2 \mathrm{ng} / \mathrm{ml}$ ). The unconjugated anti-CRP antibody was also assayed and used as negative control. The coating step was performed for 1 hour at $37^{\circ} \mathrm{C}$. After washing steps with borate buffer (3 times with $100 \mu \mathrm{l}$ using an automatic washer from BioRad), a secondary antibody conjugated with alkaline phosphatase (diluted 1:5000 in HSCD7, $50 \mu \mathrm{l} /$ well) was then incubated for 1 hour at $37^{\circ} \mathrm{C}$. After a last washing step performed as previously described, $50 \mu 1$ of AttoPhos ${ }^{\circledR}$ AP Fluorescent Substrate (Promega) was added to each well and the plate was immediately read by fluorescence (excitation $444 \mathrm{~nm}$, emission $555 \mathrm{~nm}$ ) in kinetic mode (each 2 minutes during 10 minutes) using a SpectraMax Gemini XS microplate reader (Molecular Device). Blanks (corresponding to $0 \mathrm{ng} / \mathrm{ml}$ of coated biotinylated anti-CRP antibody) were also assayed and defined as the noise. Results were calculated as signal to noise ratio.

\section{Human CRP recognition}

ELISA was performed on commercially available non-treated 96-well plates. $50 \mu 1$ of antigen (recombinant human CRP) diluted in carbonate buffer at seven different concentrations (500, $167,56,19,6,2$ and $0.7 \mathrm{ng} / \mathrm{ml}$ ) were assayed and incubated 1 hour at $37^{\circ} \mathrm{C}$. A washing step with PBS-2\%BSA was then performed (3 x $100 \mu \mathrm{l})$ using an automatic washer (Bio-Rad). Non-specific sites were then blocked with PBS-2\%BSA during 2 hours at $37^{\circ} \mathrm{C}$. Wells were 
then emptied and $50 \mu 1$ of biotinylated anti-CRP antibody was added $(0.5 \mu \mathrm{g} / \mathrm{ml}$ in PBS-2\% BSA) and incubated 1 hour at $37^{\circ} \mathrm{C}$. As negative controls some wells were also incubated with the buffer only. Another washing step (as describe before) was then performed and $50 \mu 1$ of streptavidin conjugated with alkaline phosphatase (diluted 1:5000 in PBS-2\%BSA) was added and incubated 1 hour at $37^{\circ} \mathrm{C}$. After a last washing step as describe before, $50 \mu \mathrm{l}$ of AttoPhos ${ }^{\circledR}$ AP Fluorescent Substrate was added to each well and the plate was immediately read by fluorescence (excitation 444nm, emission 555nm) in kinetic mode (each 2 minutes during 10 minutes) using a SpectraMax Gemini XS microplate reader (Molecular Device). Blanks (corresponding to $0 \mathrm{ng} / \mathrm{ml}$ of coated recombinant CRP) were also assayed and defined as the noise. Results were calculated as signal to noise ratio.

\section{Biosensor}

Optical waveguide lightmode spectroscopy (OWLS) is based on grating-assisted coupling of laser light into an optical waveguide layer. For the OWLS measurements, planar optical waveguides $(8 \times 12 \mathrm{~mm})$ made of a pyrolized $\mathrm{SiO}_{2}-\mathrm{TiO}_{2}$ sol-gel waveguiding layer were used in a BIOS-1 instrument (MicroVacuum Ltd., Hungary). The prepared waveguides were mounted in a microfluidic assembly equipped with a septum, and the assembly was mounted on the goniometer of the BIOS-1 system [8]. The reagent flow was maintained at $1 \mu 1 / \mathrm{s}$ in the flowcell by a peristaltic pump (Ismatec, Reglo). The biotinylated anti-CRP antibody and the recombinant CRP solutions were injected by a Hamilton syringe $(50 \mu \mathrm{l})$ through the septum which was closely mounted to the flowcell. The refractive index of the solutions was measured by a precision refractometer (J157 Automatic refractometer, Rudolph).

\section{Protocol used for the OWLS chip off-line PEI coating and biotinylation:}

1. Cleaning of the OWLS chips by chromosulphuric acid using a standard protocol of the manufacturer (MicroVacuum Ltd., Hungary).

2. Coating of the chip surface by PEI. Incubation of the $0.5 \%$ PEI solution $(\mathrm{pH} 9.5$ borate buffer) in an open cuvette designed in the workshop for this off-line preparation step, 20 minutes.

3. Rinse with borate buffer.

4. Cross-linking of the deposited PEI layer by $2.5 \%$ glutaraldehyde solution (in borate buffer), 2 hours.

5. Rinse with phosphate buffer.

6. Biotinylation of the PEI layer by NHS-biotin. Working solution is prepared in $20 \mathrm{mM}$ phosphate buffer at $1 \mathrm{mg} / \mathrm{ml}$ NHS-biotin, and incubate the chip for 2 hours.

7. Rinse and store in phosphate buffer until use. In these experiments the functionalized surfaces were stored up to 3 days before the OWLS measurement. 


\section{Results and discussion}

\section{ELISA results on immuno-reagents}

The biotinylated anti-CRP antibody and the recombinant CRP were evaluated by two ELISA approaches. As presented in Figure 2, the functional biotinylation of the anti-CRP antibody was characterized by assaying different concentrations of the antibody onto a neutravidin surface. Indeed a decrease of signal associated with the decreasing concentration of the coated biotinylated antibody was observed. In the same time no signal were observed with the unconjugated antibody. The affinity of the biotinylated antibody with its corresponding target protein (i.e. human recombinant CRP) was evaluated by direct ELISA. Figure 2 showed the decrease of signal associated with the reduced amount of recombinant CRP coated onto the surface of the plate wells. For this condition, limit of detection (LOD) was arbitrarily considered to be at $0.7 \mathrm{ng} / \mathrm{ml}$ (It corresponds to the lowest detected concentration of coated recombinant CRP).

\section{Figure 2.}

The PEI surface layer in previous publications has been mainly used as a promoter, rendering a positively charged surface in a simple way for further surface studies. The ideal hyperbranched interface structure on the sensor surface should be designed by tuning the deposition parameters; one of the most important of these is the $\mathrm{pH}$ of the solution. It is reported that at higher $\mathrm{pH}$ the deposited mass of PEI is higher. When the $\mathrm{pH}$ decreased, the globular molecule conformation becomes increasingly flat [21]. This was the main reason to use $\mathrm{pH} 9.5$ borate buffer during PEI deposition which is near to the $\mathrm{pK}_{\alpha}$ of PEI $(\sim 10.5)$. Our intention was to keep the thick, branched structure on the surface to obtain a high density of active points for further grafting. In the first step, the OWLS chip surface was coated off-line (in a home made cuvette) with $0.5 \%$ PEI solution in borate buffer $\mathrm{pH} 9.5$ for 20 minutes.

The question of the adsorbed PEI layer stability can be raised when the borate buffer ( $\mathrm{pH} 9.5$ ) is replaced by phosphate buffered saline (PBS, $\mathrm{pH}$ 7.4) which is normally used in bioassays and then following rinse with other solution (e.g. for regeneration). Glutaraldehyde was used to crosslink the surface adsorbed PEI molecules in order to make the PEI layer robust and durable. During the control experiments the PEI deposition was followed online with the subsequent glutaraldehyde crosslinking and buffer change. In these experiments first a 
baseline was obtained with borate buffer, then $0.5 \%$ PEI in borate, $2.5 \%$ glutaraldehyde in borate were subsequently injected, and finally washing with borate and PBS buffers. The last washing step shows specifically the possible washing out effect caused by the buffer exchange (Figure 3). These results confirm that a stable interface layer has been built up from the PEI, as there was no loss observed after repeated washing with borate buffer and PBS.

The so-called 4-layer optical model resulted in thickness of the crosslinked PEI layer of 5-10 $\mathrm{nm}$ [22]. For the given experiment in Fig. 3 the thickness $(\sim 7 \mathrm{~nm}$ in PBS) shows that the deposition parameters resulted in a relatively thick PEI layer. This range of thickness (5-10 $\mathrm{nm}$ ) is obviously larger than the quasi monomolecular thickness of silanes and thus very suitable for biomolecule immobilization. Our results show however that the highly extended molecular configuration of PEI from the surface is not probable as was depicted in [20]. The surface mass density of deposited PEI layer determined from these OWLS measurements is about $0.8 \mu \mathrm{g} / \mathrm{cm}^{2}$ which is in agreement with previously published results for silica surfaces [23]. It is noted that the apparent thickness and mass of PEI contains the contribution from the glutaraldehyde. Taking into account the large difference between the molecular masses, this contribution is almost negligible. Varga and co-workers demonstrated [24] that the PEI adsorption layers are not homogeneous but can be considered as consisting of a compact inner layer and a dilute and thicker outer layer. Our results are in agreement with this study with a slightly larger PEI layer thickness ${ }^{1}$. As the $5-10 \mathrm{~nm}$ is an average value for the thickness (from the homogeneous and isotropic model) we argue that the diluted outer PEI layer shall be farther from the surface.

\section{Figure 3.}

\section{Ex-situ surface preparation (PEI coating and biotinylation), and in situ CRP assay}

The PEI-based immobilization was demonstrated on the CRP label free assay as a part of our main project (FP7-ICT4-P3SENS, see also [25]). The OWLS chips were functionalized with PEI ex-situ with the parameters obtained previously. After the PEI deposition, the chip surface was rinsed with borate buffer and biotinylated with $1 \mathrm{mg} / \mathrm{ml} \mathrm{NHS-biotin} \mathrm{ex-situ} \mathrm{as}$ described in the protocol (Materials and Methods). The biotinylation parameters were chosen from previous experiences and publication [26]. After this surface preparation the biosensor chips were measured in the flow-cell of the OWLS system. The flow rate was set to $1 \mu 1 / \mathrm{s}$ by

\footnotetext{
${ }^{1}$ It should also be noted that our study has been performed on $\mathrm{Si} / \mathrm{TiO}_{2}$ sol-gel waveguide surface, and the PEI deposition parameters ( $\mathrm{pH}$ and crosslinking) were also slightly different from the experiment in [24].
} 
means of a peristaltic pump. First, $50 \mu \mathrm{g} / \mathrm{ml}$ neutravidin solution (in PBS) was injected into the flow cell and exposed to the biotinylated surface. Upon introduction of the biotinylated anti-CRP antibody, a fast and large increase in the signal was normally observed (as a consequence of the higher refractive index of the reagent solution), and a significant decrease was found during PBS washing due to the same reason (see Figure 4).

It is generally accepted and also reported in the OWLS literature that the determined optogeometrical parameters (thickness and refractive index) of the surface deposited biomolecular layers are often unrealistic [27]. This is in connection with the fact that in several cases the layers are anisotropic or/and inhomogeneous. The homogeneous and isotropic adlayer model obviously fails to model these films. However, the determined mass value is acceptable due to the compensation of the errors in layer thickness and refractive index. Therefore, the layer thicknesses can be estimated from the calculated mass values using an averaged refractive index $(n=1.45)$ for the protein film [28] and the well-known Feijter formula $\left(\mathrm{d}[\mathrm{nm}] \cong 15.38 \times \mathrm{M}\left[\mu \mathrm{g} / \mathrm{cm}^{2}\right]\right)$. Using this method we calculated that the PEI layer has an averaged thickness of around $5-10 \mathrm{~nm}$ and this is further increased by around 6-12 $\mathrm{nm}$ when the subsequent molecular layers are deposited. Anisotropy evaluation is planned to assess this effect on the adlayer thickness calculation.

It is noted that $0.05 \%$ Tween 20 in PBS (TPBS) was included in the final washing steps after the recombinant CRP binding to remove any possible non-specifically bound molecules from the sensor chip (Figure 4.). There was a clear detection of $10 \mu \mathrm{g} / \mathrm{ml}$ recombinant CRP, giving a detected mass of $0.024 \mu \mathrm{g} / \mathrm{cm}^{2}$ on the OWLS chip surface. Calculating the noise level from the PBS line of lower concentration data (Fig. 4.b), we can estimate that the detection limit to be about $0.2 \mu \mathrm{g} / \mathrm{mL}$ for the direct binding assay (based on the generally accepted $3 \sigma$ value). Using a more sensitive optical sensor, such as the grating coupled interferometry (GCI) [29], this detection limit could be further improved. As a negative control, another protein (NeuronSpecific Enolase; NSE) was tested; the functionalized surface with biotinylated anti-CRP antibody did not bind NSE while proved the reactivity towards the corresponding antigen (CRP).

\section{Figure 4.}

\section{Conclusion}

The idea was proposed with PEI surface functionalization to graft receptor IgG molecules through the specific biotin-neutravidin-biotin linkage. The PEI layer was crosslinked by 
glutaraldehyde to avoid washing away from the surface. At higher $\mathrm{pH}$, the adsorbed PEI layer is more extended, exposing free amino groups that can be used for immobilization. An advantageous property of such PEI layer is the flexibility and hydrophilicity of these extended molecule loops. The PEI layer can be further developed as a competitive method of immobilization, having the advantage of simplicity (possible requires 1-2 steps), relatively low cost using aqueous solutions, there is no need for thermal curing (as it is the case for silane chemistry) neither plasma treatment. The number of available free amino groups will serve as convenient points for further chemical modification. The stability of the glutaraldehyde-crosslinked PEI layer has been proved. The PEI based immobilization was demonstrated with CRP sensing using a label free, direct binding assay format.

\section{Acknowledgement}

This work was financed by the European Commission through the seventh framework program FP7-ICT4-P3SENS (248304). This work was also supported by the Lendület program of the Hungarian Academy of Sciences. RH gratefully acknowledges the Reintegration Marie Curie Fellowship from the European Commission (OPTIBIO) and the Fellowship from the Hungarian Scientific Research Fund (OTKA PD). This work was supported by the National Development Agency grants TÁMOP-4.2.2/B-10/1-2010-0025. The authors would like to gratefully thank the instrumental support of Prof. U. Bilitewski, Prof. M. Textor and Prof. J. Vörös. 


\section{References}

[1] M.C. Estevez, M. Alvarez, L.M. Lechuga, Integrated optical devices for lab-on-a-chip biosensing applications, Laser Photonics Rev. 6(4) (2012) 463-487.

[2] R. Polzius, Th. Schneider, F.F. Bier, U. Bilitewski, W. Koschinski, Optimization of biosensing using grating couplers: immobilization on tantalum oxide waveguides, Biosens. Bioelectron. 11(5) (1996) 503-514.

[3] G.T. Hermanson, Bioconjugate Techniques, Elsevier, San Diego, 1996.

[4] S. Löfas, B. Johnsson, A novel hydrogel matrix on gold surfaces in surface plasmon resonance sensors for fast and efficient covalent immobilization of ligands, J. Chem. Soc. Chem. Commun. 21 (1990) 1526-1528.

[5] F. Dortu, H. Egger, K. Kolari, T. Haatainen, P. Furjes, Z. Fekete, D. Bernier, G. Sharp, B. Lahiri, S. Kurunczi, J.C. Sanchez, N. Turck, P. Petrik, D. Patko, R. Horvath, S. Eiden, T. Aalto, S. Watts, N.P. Johnson, R.M. De La Rue, D. Giannone, Design and process development of a photonic crystal polymer biosensor for point of care diagnostics, Proc. of SPIE-OSA Biomedical Optics 8087 (2011) 80870D-1-17.

[6] D. Giannone, F. Dortu, D. Bernier, N. Johnson, G.J. Sharp, L. Hou, A.Z. Khokhar, P. Fürjes, S. Kurunczi, P. Petrik, R. Horvath, T. Aalto, K. Kolari, S. Ylinen, T. Haatainen, H. Egger, NIL fabrication of a polymer based photonic sensor device in P3SENS project, Proc. of SPIE 8435 (2012) 843529-1-8.

[7] F. Höök, J. Vörös, M. Rodahl, R. Kurrat, P. Böni, J.J. Ramsden, M. Textor, N.D. Spencer, P. Tengvall, J. Gold, B. Kasemo, A comparative study of protein adsorption on titanium oxide surfaces using in situ ellipsometry, optical waveguide lightmode spectroscopy, and quartz crystal microbalance/dissipation, Colloids Surf. B 24 (2002) 155-170.

[8] J. Vörös, J.J. Ramsden, G. Csúcs, I. Szendrő, S.M. De Paul, M. Textor, N.D. Spencer, Optical grating coupler biosensors, Biomaterials, 23 (2002) 3699-3710.

[9] R. Horvath, G. Fricsovszky, E. Papp, Biosens. Bioelectron.18 (2003) 415-428. 
[10] R. Horvath, H.C. Pedersen, F.J.G. Cusinier, Guided wave sensing of polyelectrolyte multilayers, Appl. Phys. Lett., 88 (2006) 111102-1-3.

[11] Ph. Lavalle, C. Gergely, F. J. G. Cuisinier, G. Decher, P. Schaaf, J. C. Voegel, C. Picart, Comparison of the Structure of Polyelectrolyte Multilayer Films Exhibiting a Linear and an Exponential Growth Regime: An in Situ Atomic Force Microscopy Study, Macromolecules, 35 (2002) 4458-4465.

[12] R.W.F. Wiertz, E. Marani, W.L.C. Rutten, Neural cell-cell and cell-substrate adhesion through N-cadherin, N-CAM and L1, J. Neural. Eng., 8 (2011) 046004

[13] N. Trummer, N. Adányi, M. Váradi, I. Szendrő, Modification of the surface of integrated optical wave-guide sensors for immunosensor applications, Fresenius J. Anal. Chem. 371 (2001) 21-24.

[14] S. Kurunczi, R. Horvath, Y.P. Yeh, A. Muskotál, A. Sebestyén, F. Vonderviszt, J.J. Ramsden, Self assembly of rodlike receptors from bulk solutions, J. Chem. Phys. 130 (2009) 011101

[15] R. Horvath, H. C. Pedersen, N. Skivesen, C. Svanberg, N.B. Larsen, Fabrication of reverse symmetry polymer waveguide sensor chips on nanoporous substrates using dipfloating, J. Micromech. Microeng. 15 (2005) 1260-1264.

[16] M. Wilchek, E.A. Bayer, Avidin-biotin mediated immunoassays: overview, Methods Enzymol. 184 (1990) 467-469.

[17] C. Grunwald, A biref introduction to the streptavidin-biotin system and its usage in modern surface based assays, Z. Phys. Chem. 222 (2008) 789-821.

[18] E.M. Macy, T.E. Hayes, R.P. Tracy, Variability in the measurement of C-reactive protein in healthy subjects: implications for reference interval and epidemiological applications. Clin. Chem. 43(1) (1997) 52-58.

[19] S.H. Jung, J.W. Jung, I.B. Suh, J.S. Yuk, W.J. Kim, E.Y. Choi, Y.M. Kim, K.S. Ha, Analysis of C-Reactive Protein on Amide-Linked N-Hydroxysuccinimide-Dextran Arrays with a Spectral Surface Plasmon Resonance Biosensor for Serodiagnosis, Anal.Chem. 79 (2007) 5703-5710. 
[20] J. Anzai, M. Nishimura, Layer-by-layer deposition of avidin and polymers on a solid surface to prepare thin films: significant effects of molecular geometry of the polymers on the deposition behaviour, J. Chem. Soc. Perkin Trans. 2 (1997) 1887-1889.

[21] R. Mészáros, L. Thompson, I. Varga, T. Gilányi, Adsorption properties of polyethyleneimine on silica surfaces in the presence of sodium dodecyl sulfate, Langmuir 19 (2003) 9977-9980.

[22]T. Tiefenthaler, W. Lukosz, Sensitivity of grating couplers as integrated-optical chemical sensors, J. Opt. Soc. Am. B 6 (1989) 209-220

[23] R. Mészáros, I. Varga, T. Gilányi, Adsorption of poly(ethyleneimine) on silica surfaces: effect of pH on the reversibility of adsorption, Langmuir 20 (2004) 5026-5029.

[24] I. Varga, A. Mezei, R. Mészáros, P.M. Claesson, Controlling the interaction of poly(ethylene imine) adsorption layers with oppositely charged surfactant by tuning the structure of the preadsorbed polyelectrolyte layer, Soft Matter 7 (2011) 10701-10712.

[25] http://www.p3sens-project.eu/ (28-Nov-2012)

[26] M.J. Swan, L.L. Peel, S. Carrington, N.J. Freeman, Dual-polarization interferometry: an analytical technique to measure changes in protein structure in real time, to determine the stoichiometry of binding events, and to differentiate between specific and nonspecific interactions, Anal. Biochem. 329 (2004) 190-198.

[27] R. Horvath, J.J. Ramsden, Quasi-isotropic Analysis of Anisotropic Thin Films on Optical Waveguides, Langmuir 23 (2007) 9330-9334.

[28] J. Vörös, The Density and Refractive Index of Adsorbing Protein Layers, Biophys. J. 87 (2004) 553-561.

[29] P. Kozma, A. Hámori, S. Kurunczi, K. Cottier, R. Horvath, Grating coupled optical waveguide interferometer for label-free biosensing, Sens. Actuators B 155 (2011) 446-450. 


\section{Figure captions:}

Figure 1. Schematic view of the proposed OWLS biochip surface showing the molecular structure for immobilization of $\mathrm{IgG}$. The method uses biotin-avidin interaction on a hyperbranched polymeric PEI interface layer.

Figure 2. Signal to noise ratio obtained for the two ELISA approaches.

Figure 3. On-line OWLS experiment for in situ PEI layer deposition on the chip surface from borate buffer ( $\mathrm{pH}$ 9.5), and washing test by buffer exchange to PBS (pH 7.4). Experimental data is presented as the effective refractive index, $\mathrm{N}(\mathrm{TE}+)$, of the adlayer. (B. denotes Borate buffer)

Figure 4. CRP measurement on OWLS chip using the PEI-based immobilization. A: 10 $\mu \mathrm{g} / \mathrm{ml} \mathrm{CRP}$ concentration, B: $1 \mu \mathrm{g} / \mathrm{ml}$ CRP concentration 
Figure 1.

(Color on the Web only, the printed version should be in greyscale.)

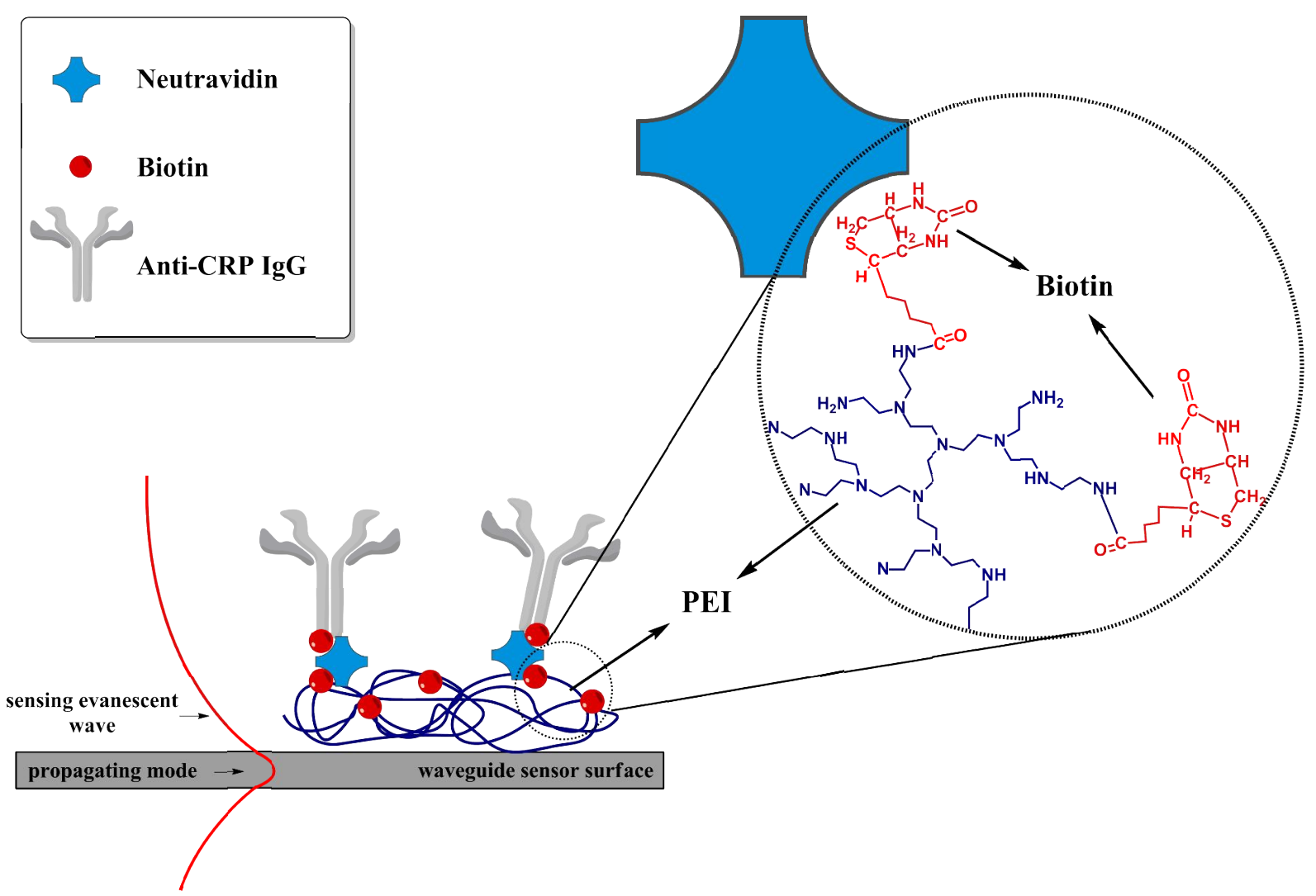


Figure 2.

(Color on the Web only, the printed version should be in greyscale.)

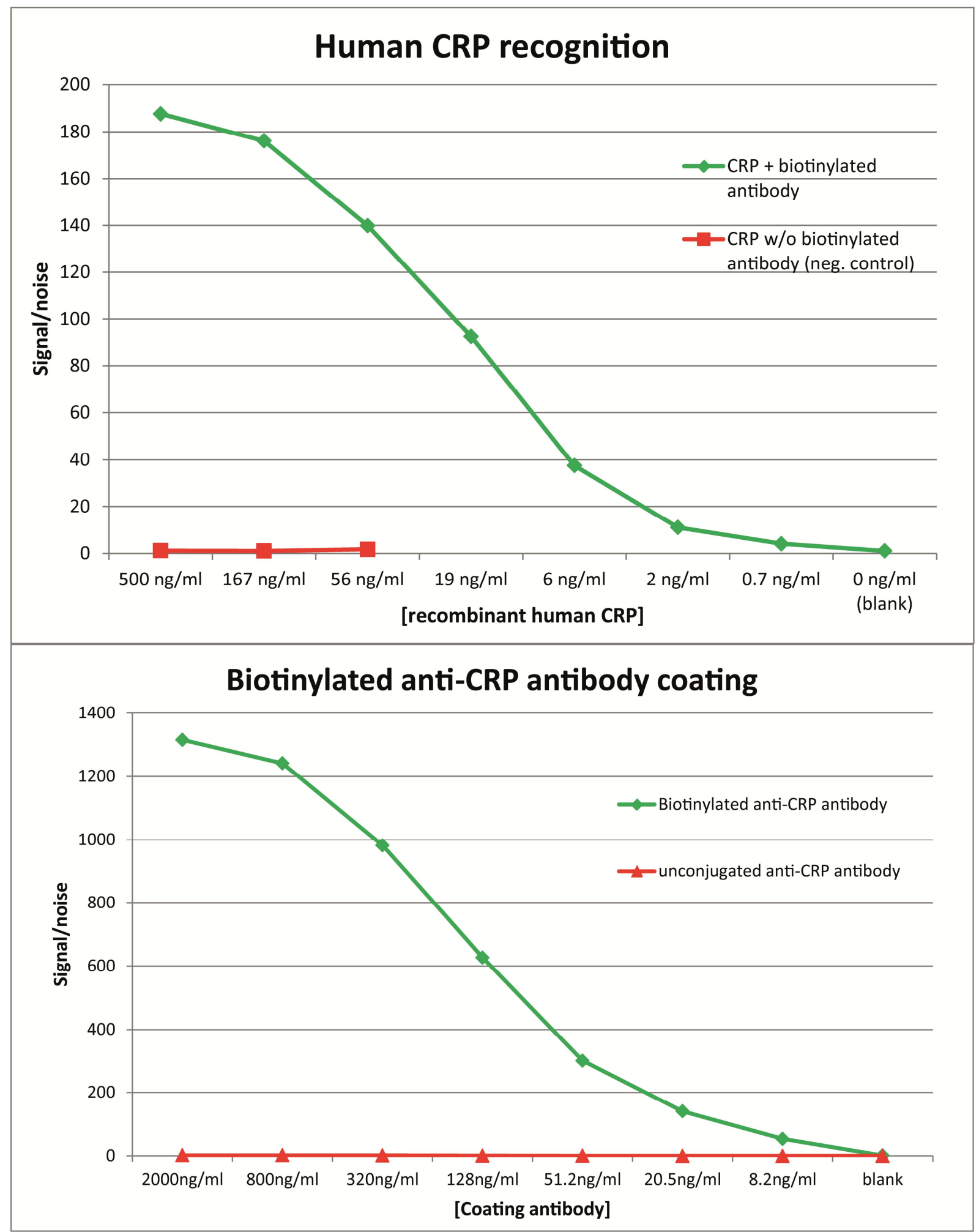


Figure 3.

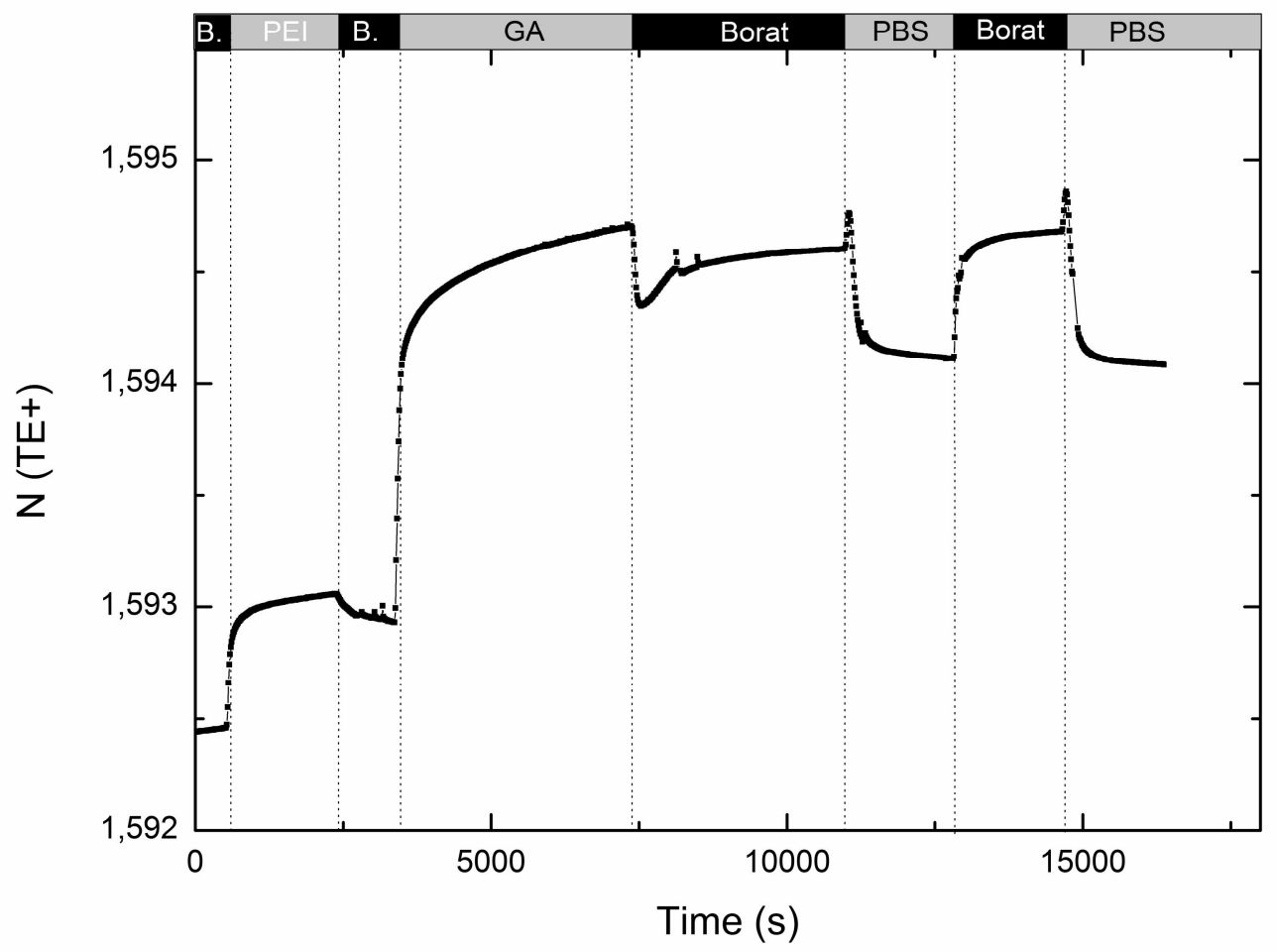


Figure 4.
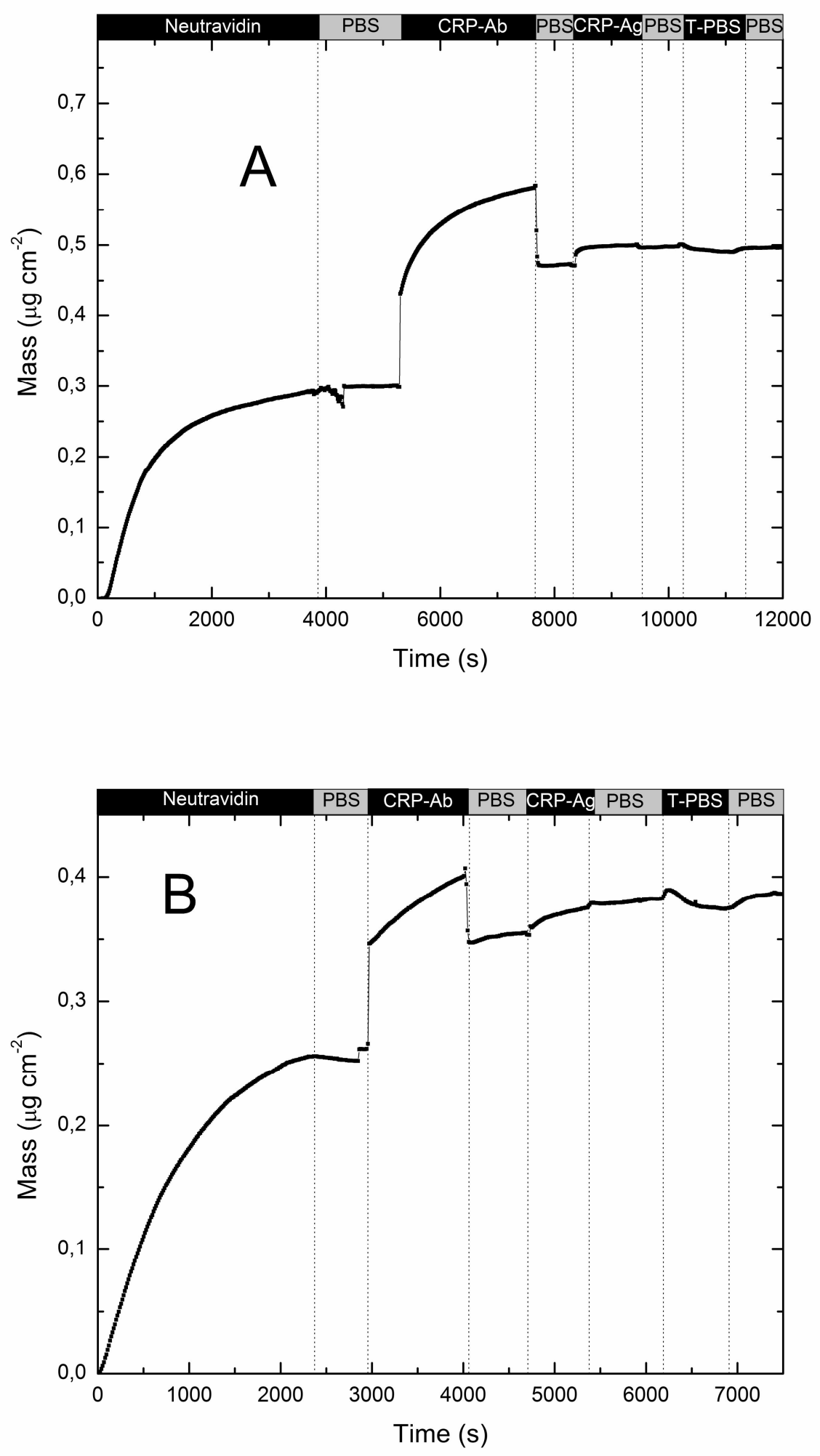